\title{
¿MARCAR O SUPERAR FRONTERAS? ESTUDIO DE CONSTRUCCIÓN DE IDENTIDAD DE PROFESORES COLOMBIANOS EN ESCUELAS PÚBLICAS DE LOS ESTADOS UNIDOS
}

\author{
Eliana Garzón DUARTE
}

Universidad de Szeged

\begin{abstract}
Resumen: Teniendo en cuenta el marco teórico del presente coloquio sobre Américas Transnacionales, esta ponencia busca explicar cómo las jerarquías lingüísticas y las ideologías del lenguaje están involucradas en la indexicalidad de espacios de pertenencia de los hablantes y cómo a partir del uso de la palabra "nosotros" se marcan fronteras sociales e ideológicas alrededor de los seres humanos. Para esto se realizó un estudio a menor escala con profesores colombianos que se encuentran vinculados laboralmente con escuelas públicas en los Estados Unidos. Los profesores respondieron un cuestionario en línea y participaron de una entrevista por Skype. Los resultados se analizaron teniendo en cuenta el principio de indexicalidad dentro del estudio de identidad, propuesto por Bucholtz y Hall (2010). Los estudios de discurso contemporáneos argumentan que las identidades sociales no son fijas, sino que se construyen de acuerdo al posicionamiento que el hablante busca habitar en el curso de una interacción lingüística para alcanzar una meta específica en las negociaciones de pertenencia con los demás. De aquí que el concepto de frontera tampoco sea fijo, sino que se entiende como una construcción simbólica que se funda en una subjetividad que circunscribe al mundo del "uno" y el “otro”, desde las prácticas culturales, sociales y lingüísticas.
\end{abstract}

Palabras clave: indexicalidad, identidad, frontera, pertenencia, jerarquías lingüísticas.

\begin{abstract}
In the framework of the present colloquium on Transnational Americas, this talk aims to explain how language ideologies and linguistic hierarchies are involved in indexing speakers to spaces of belonging that draw ideological and social boundaries around human beings from the use of the word "we". With this purpose, a small-scale study with Colombian teachers in public schools of USA was carried out. These teachers answered an online questionnaire and participated in an interview via Skype. The results were analyzed taking into account the principle of indexicality, proposed by Bucholtz and Hall (2010). Contemporary discourse studies have shown that social identities are not fixed, but constructed according to the positioning speakers seek to inhabit temporarily in the course of a linguistic interaction to achieve specific social goals in the negotiation of belonging with the others. Thus the concept of boundary is not either fixed, but it is understood as a symbolic construction that is founded on a subjectivity that circumscribes the world of "one" and "other", from the cultural, social and linguistic practices.
\end{abstract}

Keywords: indexicality, identity, border, belonging, linguistic hierarchies. 
¿Marcar o superar fronteras? Estudio de construcción de identidad de profesores colombianos en escuelas públicas de los Estados Unidos

\section{Introducción}

El presente estudio se realizó con veinte profesores colombianos entre los 26 a 43 años de edad, que se encuentran vinculados desde hace varios años a escuelas públicas de los Estados Unidos. El rango de tiempo de experiencia como profesores en los Estados Unidos oscila entre 2 a 14 años. Estos profesores lograron su vinculación laboral participando en un programa de intercambio que contrata profesores de diferentes países del mundo para laborar por un tiempo mínimo de 3 años. Algunos profesores logran extender su contrato laboral de acuerdo a los resultados de su desempeño y algunos de ellos inician su trámite de residencia en los Estados Unidos, continuando con su vinculación laboral en la escuela pública. Este programa de intercambio permite que los profesores cursen estudios de maestría mientras se encuentran vinculados laboralmente a la escuela. Los estados que participan en este programa de intercambio de profesores internacionales son Carolina del Norte, Carolina del Sur, Texas y Virginia. Sin embargo, dentro del grupo de participantes en el presente estudio, se encuentran también profesores que laboran en escuelas públicas de Nueva York.

Los profesores participantes respondieron un cuestionario en línea y algunos de ellos participaron en una entrevista vía Skype, para complementar la información suministrada en el cuestionario. Los temas que se abordaron en el estudio fueron las razones por las cuales decidieron trabajar en los Estados Unidos; la forma como ellos se identifican a sí mismos en los Estados Unidos y cómo los identifican los demás; las percepciones de los padres de familia, estudiantes y colegas hacia ellos; dificultades que han enfrentado por su acento español; su actitud hacia la práctica del español en los Estados Unidos; proyecciones laborales y diferencias en la disposición de los estudiantes hacia ellos.

Las respuestas de los participantes fueron analizadas teniendo en cuenta los principios de estudio de identidad, propuestos por Bucholtz y Hall (2010), haciendo mayor énfasis en el principio de indexicalidad y la manera como los participantes van posicionándose en identidades sociales de acuerdo al contexto comunicativo y al sentido de pertenencia que quieran alcanzar en ciertas interacciones lingüísticas. En las respuestas se puede observar, de igual manera, cómo el concepto simbólico de frontera se ve trazado a partir de las ideologías del lenguaje como espacio socialmente construido y cambiante por las prácticas sociales y lingüísticas. 


\section{Identidad y lenguaje}

La identidad debe entenderse como un fenómeno sociocultural y relacional que se manifiesta en un discurso; es decir, está dinámicamente y constantemente construida y negociada por los hablantes en una interacción lingüística. La identidad no es una estructura que expresa categorías sociales fijas, sino que opera en múltiples niveles lingüísticos simultáneamente, los cuales adquieren un significado social. Bucholtz y Hall explican la identidad como "el posicionamiento social de sí mismo y del otro" (2005:586). Por eso, se hace necesario entender la conexión entre lenguaje e identidad como un elemento fundamental de nuestra experiencia como seres sociales. El lenguaje no solamente refleja quiénes somos nosotros, sino que de cierta manera es lo que somos nosotros. Según Joseph (2010), el lenguaje nos define directa e indirectamente.

Llamas y Watt (2010) argumentan cómo el lenguaje es usado de manera directa para denotar y describir quién es una persona, a través del uso de nombres y términos de parentesco, apariencia, comportamiento y origen. También, es usado de manera indirecta cuando se asignan identidades a los otros de acuerdo a su estilo de lenguaje. Ni nuestras identidades ni nuestro lenguaje son estáticos. Ambos están cambiando constantemente y se están renegociando en respuesta a los continuos contextos cambiantes de interacciones lingüisticas y sociales (MendozaDenton, 2002).

La negociación de identidad es un proceso construido socialmente. Las identidades son constantemente creadas y negociadas en un nivel personal entre el sí y el otro. Busch (2015) argumenta que las ideologías lingüísticas son usadas para construir afiliaciones y exclusiones sociales, nacionales y étnicas. Por consiguiente, el lenguaje tiene poder y afecta la manera como somos percibidos y como percibimos a los otros. La percepción de uno mismo y del otro se da y se desarrolla en la comunicación, donde las declaraciones no tienen solamente un significado denotativo, sino que implican una posición del hablante en la sociedad (Blommaert, 2005). El vínculo interpretativo entre lo que se dice y su contexto se le conoce como índice. El principio de indexicalidad hace referencia a formas lingüísticas semióticamente vinculadas a significados sociales. Las identidades son construidas o indexadas a través de formas y prácticas lingüísticas particulares y dependen en gran parte de estructuras ideológicas. 
¿Marcar o superar fronteras? Estudio de construcción de identidad de profesores colombianos en escuelas públicas de los Estados Unidos

\section{Los cinco principios para el estudio de la identidad}

Bucholtz y Hall (2010) proponen cinco principios fundamentales para el estudio de la identidad. Estos principios son el de afloramiento (emergence principle), posicionalidad (positionality), indexicalidad (indexicality), relacionalidad (relationality) y parcialidad (partialness). El primer principio aborda la identidad desde su surgimiento en condiciones concretas de interacción lingüística y no como una fuente pre-existente de prácticas semióticas o lingüísticas. El principio de posicionalidad estudia la identidad como representación de roles que surgen temporalmente en la interacción lingüística. El principio de la indexicalidad, como ya fue mencionado anteriormente, envuelve dos niveles semióticos: en el nivel directo, las formas lingüísticas son asociadas a posturas interaccionales u orientaciones al discurso en desarrollo, mientras que en el nivel indirecto, estas posturas se ligan a formas más duraderas de ser, es decir estilos o identidades, las cuales están asociadas ideológicamente a grupos sociales, como lo argumenta Ochs (1992). En el principio de la relacionalidad, las identidades nunca están aisladas, sino que ganan significado social a través de la interacción con otras identidades y con otros actores sociales. Finalmente, el principio de la parcialidad estudia la identidad desde su parte intencional y su parte habitual y no plenamente consciente. La identidad es fracturada y discontinua siempre localizada en un contexto discursivo.

\section{Análisis de datos}

El análisis recurre a un tipo de datos que emerge de las respuestas de los profesores participantes en cuanto a su experiencia como colombianos y hablantes de español en los Estados Unidos. En estas respuestas se estudia el nivel directo de indexicalidad del acento español latino en el discurso oral en inglés de los colombianos y el nivel indirecto de indexicalidad del acento español latino asociado ideológicamente a la inmigración ilegal en los Estados Unidos. Dentro de este último nivel de indexicalidad, el concepto de frontera como espacio simbólico socialmente construido por las prácticas sociales y lingüísticas, es evidente en las experiencias de interacción lingüística de los participantes en los diferentes contextos comunicativos en el territorio de los Estados Unidos.

Una de las experiencias narradas por uno de los profesores participantes muestra la percepción de los otros ante su acento español latino al hablar en inglés: 
Cuando fui a renovar mi Green Card, el empleado de la oficina de recepción de documentos me hablaba lentamente, utilizando sus manos y expresión facial para hacerme entender lo que estaba diciendo. Vocalizaba cada palabra muy despacio e iba imitando con las manos lo que yo debía hacer para la siguiente cita. Cuando vio que mi esposo era horticultor, le dijo en tono de ironía que yo podía enseñarle a cultivar plantas de marihuana en la casa (Q.1-P.2Jessi).

En esta respuesta, se puede observar que la percepción del empleado americano al identificar un acento diferente al inglés nativo es la de asociar a la hablante con falta de conocimiento del lenguaje inglés. Las jerarquías lingüísticas hacen que la hablante sea identificada como una inmigrante, como alguien que no pertenece al espacio donde se desarrolla la interacción. Esta experiencia evidencia precisamente el concepto de frontera y de no pertenencia al espacio que la hablante quiere habitar. Todo esto marcado específicamente por el lenguaje y por la identidad social que trae consigo su lugar de procedencia. Otra respuesta que sustenta esta interpretación es "Cuando notan mi acento inmediatamente creen que no puedo entender lo que ellos dicen. Que no sé inglés" (Q.1-P.3-Carlos).

Algunas otras respuestas suministradas por los participantes en cuanto a experiencias vividas por su acento en los Estados Unidos, que evidencian la construcción simbólica de frontera y de no pertenencia, a partir de las características de las jerarquías lingüísticas que están inmersas en el estudio de identidad son las siguientes:

\footnotetext{
Una vez en una entrevista en una escuela, con una directora asistente que era Afro-americana tuve un problema. Ella me detuvo en medio de una de mis respuestas porque no le gustaba mi acento y agregó que no me entendía. No pude continuar con la entrevista hasta tanto otra persona vino a formularme las preguntas. Mis resultados se vieron afectados por este inconveniente (Q.1-P.2-Julián).
}

"Los estudiantes afro-americanos son más reacios a los profesores con acento hispánico. Siempre tratan de tomar ventaja por nuestro acento y empiezan a usar slang para que no les entendamos" (Q.1-P.5-Rochi).

"He considerado la posibilidad de regresar a Colombia cuando me pensione. Antes no porque no creo que vaya a tener allá el mismo estilo de vida que tengo aquî" (E.1-P.4-Fernando). 
¿Marcar o superar fronteras? Estudio de construcción de identidad de profesores colombianos en escuelas públicas de los Estados Unidos

"Quiero regresar a Colombia y conseguir mejores oportunidades laborales con mi experiencia aquí en Estados Unidos. Me gusta trabajar en universidades, pero este empleo como profesor de escuela es el precio que debo pagar por mi estadía aquí” (Q.1-P.6-Diana).

Teniendo en cuenta el principio de posicionalidad, los profesores van construyendo identidades de acuerdo a los roles sociales que buscan habitar en contextos de interacción lingüística específicos. Así, no siempre se identifican ellos mismos como profesores colombianos en los Estados Unidos, sino que algunas veces se identifican como aprendices de inglés o como ciudadanos comunes, dependiendo del caso. A pesar de que la mayoría de los participantes se identifica como colombiano y latino, otros han empezado a verse como individuos que pertenecen a los Estados Unidos y sus interacciones lingüísticas buscan dejar de lado cualquier rastro que los identifique como latinos. Por ejemplo:

\footnotetext{
No quiero trabajar en Colombia. Los profesores no tienen buen salario. No vale la pena, en mi opinión. El trabajo es muy similar al que hago aquí en Estados Unidos, pero en Colombia es con menos salario y menos beneficios. Yo trato de imitar al máximo el acento inglés porque creo que el acento me ayuda a identificarme como ciudadana americana (E.1-P.4-Milena).
}

Otro aspecto interesante de nivel de indexicalidad indirecto está dado con la asociación como mexicanos que hacen algunos americanos de cualquier persona con acento español latino. Para algunos americanos, ser latino es sinónimo de mexicano. Aquí no vale el país de origen. La asociación ideológica corresponde al grupo social de mexicanos residentes en Estados Unidos. De esta manera, son percibidos, algunas veces, los profesores colombianos en el contexto escolar, por sus estudiantes o padres de familia, o en contextos corrientes donde hay interacciones comunes del día a día.

\section{Conclusiones}

En el análisis se encontró cómo las ideologías del lenguaje y las jerarquías lingüísticas están involucradas en la indexicalidad de espacios de pertenencia. Se marcan fronteras sociales e ideológicas utilizando un "Tú no eres de nosotros" porque hablas diferente. Tienes un acento que no corresponde al "nuestro". La discriminación por el acento se ha observado en algunas experiencias narradas 
por los participantes. Dentro de estas experiencias sobresale el hecho de que los otros fingen no entender lo que los hablantes colombianos expresan, tomar el acento como excusa de algunos padres de familia para justificar el bajo rendimiento de sus hijos, hacer burla de la pronunciación de algunas palabras, entre otras.

Con este estudio se observó además cómo los profesores han construido identidades dentro del espacio de Estados Unidos para alcanzar metas sociales específicas en las negociaciones de aquellas articulaciones de pertenencia con los demás (Eckert, 2003; Bucholtz y Hall, 2010). Aquí, las variables lingüísticas ganan su fuerza semiótica en contextos culturales a través de la asociación indexical con grupos sociales particulares, una asociación que puede ser forjada a través de la ideología, la práctica habitual o ambas (Silverstein, 2003).

\section{Bibliografía}

Blommaert, Jan. 2005. Discourse: A critical introduction. Cambridge, UK: Cambridge University Press.

Bucholtz, Mary y Hall, Kira. 2005. "Identity and Interaction: a Sociocultural Linguistic approach" Discourse Studies 7(4-5), 585-614.

Bucholtz, Mary y Hall, Kira. 2010. "Locating identity in language" Llamas, Carmen \& Watt, Dominic (eds.). Language and identities. Edinburgh, UK: Edinburgh University Press. 18-28.

Busch, Brigitta. 2015. "Expanding the notion of the linguistic repertoire: On the concept of Spracherleben - the lived experience of language" Applied Linguistics, 1-20.

Eckert, Penelope 2003. "The meaning of style" Texas Linguistic Forum 47, 41-53.

Joseph, John E. 2010. "Identity" Llamas, Carmen \& Watt, Dominic (eds.). Language and identities. Edinburgh, UK: Edinburgh University Press. 9-17.

Llamas, Carmen \& Watt, Dominic. 2010. "Introduction". Llamas, Carmen \& Watt, Dominic (eds.). Language and identities. Edinburgh, UK: Edinburgh University Press. 1-5.

Mendoza-Denton, Norma. 2002. "Language and identity" J. K. Chambers, Peter Trudgill, \& Natalie Schilling-Estes (eds.). The bandbook of language variation and change. Oxford: Blackwell. 475-499.

Ochs, Elinor. 1992. "Indexing gender" Duranti, Alessandro \& Goodwin, 
¿Marcar o superar fronteras? Estudio de construcción de identidad de profesores colombianos en escuelas públicas de los Estados Unidos

Charles (eds.). Rethinking context: Language as an interactive phenomenon. Cambridge: Cambridge University Press. 335-358.

Silverstein, Michael. 2003. "Indexical order and the dialectics of sociolinguistic life" Language and Communication 23(3-4), 193-229. 